\title{
LigaSure versus monopolar cautery for recipient hepatectomy in liver transplantation: a propensity score-matched analysis
}

\author{
Jeong-Moo Lee, Kwangpyo Hong^, Eui Soo Han^, Sanggyun Suh, Suyoung Hong, Suk Kyun Hong^, \\ YoungRok Choi^ ${ }^{\wedge}$, Nam-Joon Yi^, Kwang-Woong Lee ${ }^{\wedge}$, Kyung-Suk Suh^^ \\ Department of Surgery, Seoul National University Hospital, Seoul, Korea \\ Contributions: (I) Conception and design: JM Lee; (II) Administrative support: JM Lee, NJ Yi, KS Suh; (III) Provision of study materials or patients: \\ JM Lee; (IV) Collection and assembly of data: S Suh, S Hong; (V) Data analysis and interpretation: JM Lee, K Hong, ES Han; (VI) Manuscript \\ writing: All authors; (VII) Final approval of manuscript: All authors. \\ Correspondence to: Jeong-Moo Lee, MD. Assistant Professor, Department of Surgery, Seoul National University Hospital, 03080 Daehak-ro, Seoul, \\ Korea. Email: lulu5050@naver.com; jmleetpl@gmail.com.
}

\begin{abstract}
Background: Recipient hepatectomy during liver transplantation (LT) is one of the most challenging aspects of surgery due to the possibility of massive bleeding. This study aimed to compare and analyze the effectiveness between LigaSure and monopolar cautery in recipients.

Methods: We reviewed 187 recipients who underwent LT from March 2019 to June 2020. We compared the surgical outcomes of the 69 recipients who underwent recipient hepatectomy with LigaSure (LigaSure group) and 118 recipients who underwent with monopolar cautery. Propensity score matching (PSM) was performed using the nearest-neighbor method at a ratio of 1:1 based on 14 baseline characteristics and possible factors that influence postoperative bleeding.

Results: A total of 187 adult recipients were reviewed retrospectively. In the propensity score-matched analysis, The rates of bleeding and infectious complication were significantly lower in the LigaSure group than in the monopolar cautery group $(3 / 69,4.35 \%$ versus $13 / 69,18.8 \% ; \mathrm{P}=0.015$ and $1 / 69,1.45 \%$ versus $9 / 69,13.0 \% ; \mathrm{P}=0.017)$. The length of postoperative hospital stay was shorter in the LigaSure group (mean: $23.1 \pm 16.1$ versus $39.6 \pm 58.2$ days; $\mathrm{P}=0.024)$.
\end{abstract}

Conclusions: Recipient hepatectomy with LigaSure is associated with a short hospital stay due to low reoperation rates, postoperative bleeding, and secondary infection related to bleeding.

Keywords: Liver transplantation (LT); hepatectomy postoperative hemorrhage; reoperation

Submitted Mar 19, 2021. Accepted for publication May 20, 2021.

doi: 10.21037/atm-21-1318

View this article at: https://dx.doi.org/10.21037/atm-21-1318

\section{Introduction}

Recipient hepatectomy during liver transplantation (LT) is one of the most challenging parts of LT because it had a high possibility of massive bleeding. The candidate of LT had several risk factors of intraoperative bleeding.
Hemostasis is difficult due to portal hypertension-related to liver cirrhosis, leading to the development of surrounding collateral vessels and the low levels of coagulation factors and thrombocytopenia splenomegaly (1-3). Massive intraoperative bleeding may cause other postoperative morbidity (4-6). In patients with borderline cardiovascular

^ ORCID: Kwangpyo Hong, 0000-0001-7909-8116; Eui Soo Han, 0000-0001-9582-1255; Suk-Kyun Hong, 0000-0002-0020-6215; YoungRok Choi, 0000-0003-2408-7086; Nam-Joon Yi, 0000-0002-5467-425X; Kwang-Woong Lee, 0000-0001-6412-1926; Kyung-Suk Suh, 0000-0002-9535-7349. 
and renal functions, the possible postoperative complications include acute myocardial infarction and acute kidney injury.

Conversely, in patients with severe underlying diseases, hypotension related to bleeding causes vasoconstriction in the brain and heart, which results in fatal complications, such as stroke and pulmonary edema (7-9). Furthermore, the rate of secondary infectious complications due to mass transfusion and re-exploration rate would increase $(5,10)$. Therefore, the prevention of fatal intraoperative bleeding during recipient hepatectomy is key to successful LT.

With the advancements in technology related to surgical equipment, numerous surgical energy devices have been developed and are currently used in various surgery areas, such as laparoscopic surgery. Energy devices enable safe ligation of blood vessels and tissues with a sealing effect absent in the conventional monopolar cautery. A LigaSure vessel-sealing device (Medtronics, Minneapolis, MN, USA) reduces the risk of bleeding during the dissection of vessels (9,11-14). Thus, it can be beneficial for the ligation of small vessels or tissues. Several studies show the usefulness of LigaSure in gastric cancer surgery, thyroid cancer surgery, and gynecologic surgery $(12,15,16)$. Vessel-sealing devices speed up surgical procedures by reliably sealing and dividing vessels in a single action that would otherwise require multiple ligation and division steps. Furthermore, it can seal vessels without significant thermal spread, damaging the inferior vena cava (IVC).

The conventional monopolar cautery enables fast tissue dissection, and it can coagulate the ends of small-caliber vessels. But it can not deliver heat to penetrate deep into both sides of the vessels. Therefore, severe bleeding may occur when portal pressure is raised in the anhepatic phase.

The use of energy-based devices such as LigaSure and ultrasonic sear in liver surgery has been focused on laparoscopic liver parenchymal dissection. In several studies, liver parenchymal dissection takes longer than CUSA, but it has been reported that there is no difference in bleeding or surgical outcomes $(17,18)$. However, reviews on the role of energy-based devices in LT at a high risk of bleeding are still insufficient (19). This study aimed to compare LigaSure and monopolar cautery's effectiveness in recipient hepatectomy in terms of intraoperative and postoperative bleeding and complications. We present the following article in accordance with the STROBE reporting checklist (available at https://dx.doi. org/10.21037/atm-21-1318).

\section{Methods}

\section{Inclusion criteria}

This study protocol conformed to the ethical guidelines of the Declaration of Helsinki (as revised in 2013). The Institutional Review Board of Seoul National University Hospital approved this study (No.: 2101-001-1183) and individual consent for this retrospective analysis was waived. We reviewed patients who underwent LT from March 2019 to June 2020, excluding pediatric patients. A total of 187 recipients were included in this study. We compared the surgical outcomes of the 69 recipients who underwent recipient hepatectomy with LigaSure (LigaSure group) and 118 recipients who underwent with monopolar cautery. We performed Propensity score matching (PSM) using the nearest-neighbor method at a ratio of $1: 1$ based on 14 covariates. Finally, we compared surgical outcomes of 69 recipients who underwent recipient hepatectomy with LigaSure and 69 recipients who underwent with monopolar cautery.

\section{Preoperative characteristics and variables of the recipients}

We collected basic demographic data on age, gender, height, weight, body mass index, an indication of LT, and type of LT (living or deceased). Patients with hepatocellular carcinoma (HCC) were evaluated via computed tomography (CT), gadoxetic acid (Gd-EOB-DTPA, Primovist, BayerSchering Pharma, Berlin, Germany)-enhanced magnetic resonance imaging and positron emission tomography for pre-transplantation. The levels of tumor markers, such as alpha-fetoprotein (AFP) and protein induced by vitamin $\mathrm{K}$ absence II (PIVKA-II), were also evaluated. In addition, we investigated several factors thought to influence postoperative bleeding, including platelet count, prothrombin time/international normalized ratio (PT INR), presence of large varix, graft-recipient weight ratio (GRWR), previous abdominal surgery, and previous HCC treatment (transarterial chemoembolization or radiofrequency ablation).

\section{Surgical technique of recipient hepatectomy}

An inverted $\mathrm{T}$ incision (with upper midline $\mathrm{T}$ extension and xiphoid process excision) was made to enable adequate exposure of the whole abdominal field. In patients with adhesions due to previous abdominal surgery, we performed adhesiolysis with monopolar cautery or LigaSure. 

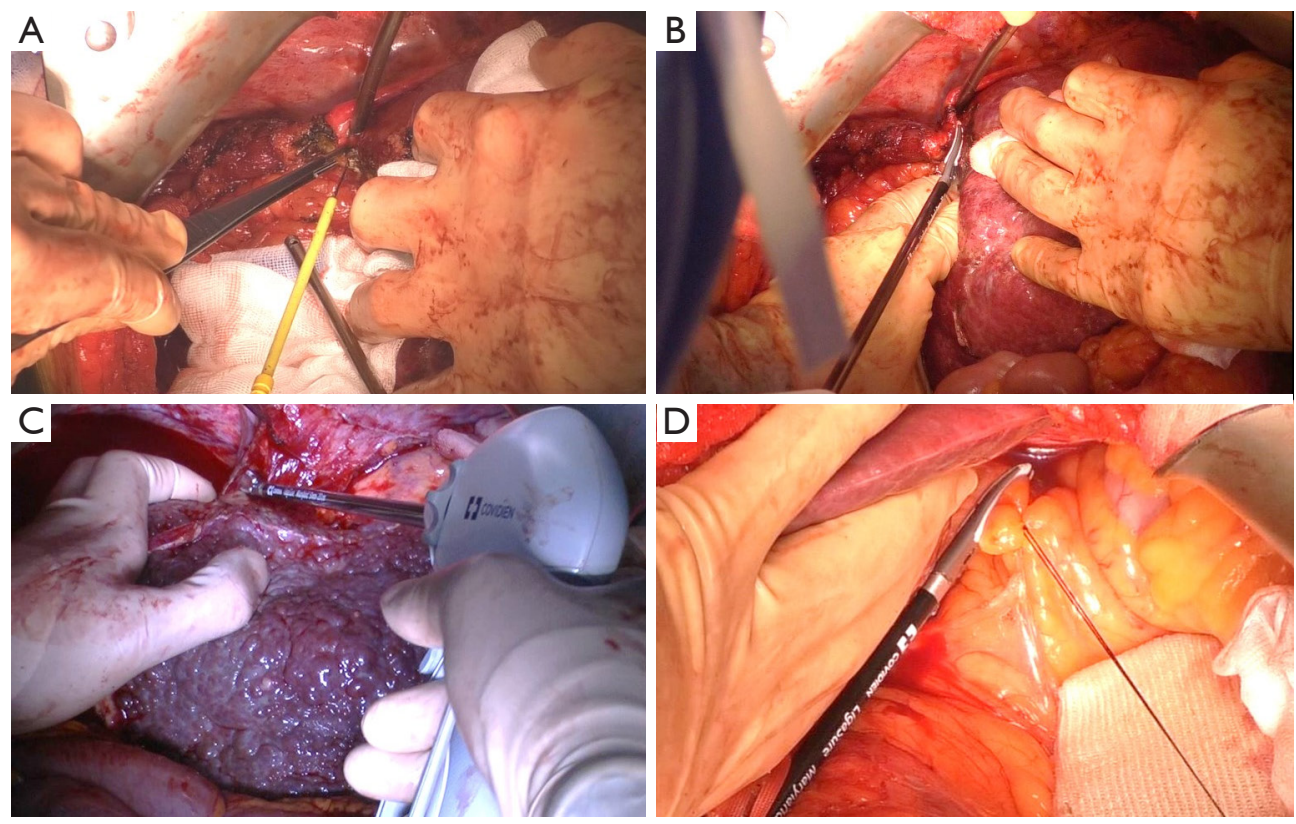

Figure 1 Clinical uses of LigaSure and monopolar cautery in the surgical field during liver transplantation. (A) Right liver mobilization using monopolar cautery. (B) Right liver mobilization using LigaSure (detachment from the diaphragm). (C) Blunt dissection around the right hepatic vein using LigaSure. (D) Ligation of the accessory left hepatic artery using LigaSure and a surgical tie.

Subsequently, we mobilized the right side of the liver and attempted to dissect the avascular space between the diaphragm and liver (Figure 1A,B). However, small varices usually develop around a cirrhotic liver, which is generally the leading cause of bleeding during recipient hepatectomy. In this procedure, monopolar cautery or LigaSure was employed (Figure 1C).

The left side of the liver was mobilized, and the left triangular ligament was divided using LigaSure or a tie. After the isolation of the hepatic hilum using nylon tape, the lesser sac between the left lateral section and caudate lobe was dissected. After the dissection, the ligamentum venosum was ligated. If an accessory left hepatic artery from the left gastric artery was present, we used the energy device and ligated the artery using a suture or a tie (Figure 1D).

The right adrenal gland is often tightly attached to the cirrhotic liver. The division of the adrenal gland causes bleeding during right liver mobilization. If the adrenal gland had a huge adrenal vein inside the parenchymal tissue, we ligated the vein directly connected to the IVC using a tie. In the monopolar cautery group, we divided the adrenal parenchyma after the dissection of the adrenal tissues. In the LigaSure group, we could split the adrenal parenchyma after blunt dissection using the energy device.
After the bilateral mobilization of the liver, the IVC was exposed. The short hepatic vein between the liver and IVC was dissected and ligated. The energy device was not used for this procedure. Instead, surgical ties or metal clips were used because of bleeding from the IVC branch in the initial periods of using the energy device. When the IVC pressure increased after transplantation, bleeding occurred from the IVC area sealed by the energy device.

The hepatic hilum was divided, and the right hepatic vein was divided after clamping using a Satinsky clamp. The recipient's liver was rotated to the left. The hepatic vein branches from the caudate lobe and retrocaval space to the IVC were ligated. The common trunk of the middle hepatic vein and left hepatic vein was divided using a vascular stapler.

\section{Intraoperative factors and postoperative outcomes}

The intraoperative factors include the total operative time, time from skin incision to liver removal, bleeding control time (after vascular anastomosis until start of wound closure), estimated blood loss volume, and the total amount of red blood cells transfused. Conversely, the postoperative factors include the length of hospital stay 
following transplantation, re-operation rate due to bleeding, and postoperative complications. The postoperative complications were graded according to the ClavienDindo classification. The postoperative laboratory findings, including liver function test, PT INR, and tacrolimus level, were measured daily until discharge. Abdominal ultrasound examination was repeated on postoperative day 6 . The Jackson-Pratt drainages were removed when abdominal dynamic CT revealed no abnormal findings and when no bile leakage occurred. In-hospital mortality was considered if the recipient died before discharge from the hospital.

\section{Statistical analysis}

PSM was performed using the nearest-neighbor method at a ratio of $1: 1$ based on 14 covariates. We matched for baseline characteristics (age, sex, height, weight, Model for End-Stage Liver Disease score, and type of LT) and factors that may influence bleeding (platelet count, PT INR, previous HCC treatment, previous abdominal surgery, previous variceal bleeding, GRWR, AFP level, and PIVKAII level). Numerical variables were expressed as mean \pm standard deviation or as median (interquartile range). The Student's $t$-test or Mann-Whitney U test was employed to compare continuous variables before PSM. Conversely, the Student's $t$-test or Wilcoxon rank-sum test was employed for continuous variables after PSM. A two-tailed $\mathrm{P}<0.05$ was considered statistically significant. All statistical analyses were conducted using the SPSS software (version 23; SPSS Inc., Chicago, IL, USA).

\section{Results}

\section{Preoperative characteristics and surgical outcomes before and after PSM (Table 1)}

A total of 187 patients underwent LT. Of these patients, 69 underwent recipient hepatectomy with LigaSure, and 118 underwent recipient hepatectomy with monopolar cautery. Before PSM, a statistically significant difference was observed in age between the LigaSure group and monopolar cautery group $(56.7 \pm 10.6$ versus $50.8 \pm 18.2$ years; $\mathrm{P}=0.006)$. Similarly, a statistically significant difference was observed in height before surgery between the LigaSure group and monopolar cautery group $(187.8 \pm 18.2$ versus $168.4 \pm 6.4 \mathrm{~cm}$; $\mathrm{P}=0.011$ ); however, no statistically significant difference was observed in other demographic characteristics between the two groups. We performed PSM using multiple logistic regression and a 1:1 matching requirement with the nearest-neighbor matching method. After PSM, no statistically significant difference was observed in the baseline characteristics before surgery between the two groups. Similarly, there was no statistically significant difference between the two groups in the factors expected to influence postoperative bleeding, such as platelet count, PT INR, history of the previous abdominal surgery, and the variceal bleeding history.

\section{Comparison of postoperative outcomes between the LigaSure group versus monopolar cautery group}

Table 2 presents the operative outcomes and postoperative hospital courses of both groups. No statistically significant difference was observed in the mean follow-up duration between the two groups $(9.94 \pm 4.12$ versus $10.7 \pm$ 4.47 months; $\mathrm{P}=0.481)$. Blood loss volume and total amount of red blood cells transfused between the two groups were not statistically significantly different between the two groups (mean: $5,486.5 \pm 9,024.6$ versus $4,754.3 \pm$ 6,819.5 mL; $\mathrm{P}=0.592$; mean: $11.01 \pm 13.75$ packs versus $10.21 \pm 12.52$ packs; $\mathrm{P}=0.447$, respectively). Moreover, there was no statistically significant difference in the total operative time between the two groups. The time from skin incision to liver removal was slightly longer in the LigaSure group than in the monopolar cautery group, but the difference between the two groups was not statically significant $(115.8 \pm 88.9$ versus $96.3 \pm 46.7, \mathrm{P}=0.111)$. The bleeding control time, which is defined as the time from anastomosis establishment to skin closure, was longer in the LigaSure group than in the monopolar cautery group (mean: $82.8 \pm 44.7$ versus $68.8 \pm 29.2 ; \mathrm{P}=0.033$ ). The length of postoperative hospital stay was shorter in the LigaSure group than in the monopolar cautery group (mean: $23.1 \pm 16.1$ versus $39.6 \pm 58.2$ days; $\mathrm{P}=0.024$ ).

The overall complication rate was significantly lower in the LigaSure group than in the monopolar cautery group (23/69, 33.3\% versus 37/69, 53.6\%; $\mathrm{P}=0.017)$. Specifically, the rates of bleeding and infectious complication were significantly lower in the LigaSure group than in the monopolar cautery group $(3 / 69,4.35 \%$ versus $13 / 69$, $18.8 \% ; \mathrm{P}=0.015$ and $1 / 69,1.45 \%$ versus $9 / 69,13.0 \%$; $\mathrm{P}=0.017$, respectively). Contrarily, no significant differences were observed in the incidence of other complications between the two groups. The rate of major complications, defined as grade III or higher according to the ClavienDindo classification, was significantly less in the LigaSure 
Table 1 Preoperative characteristics before and after propensity score matching

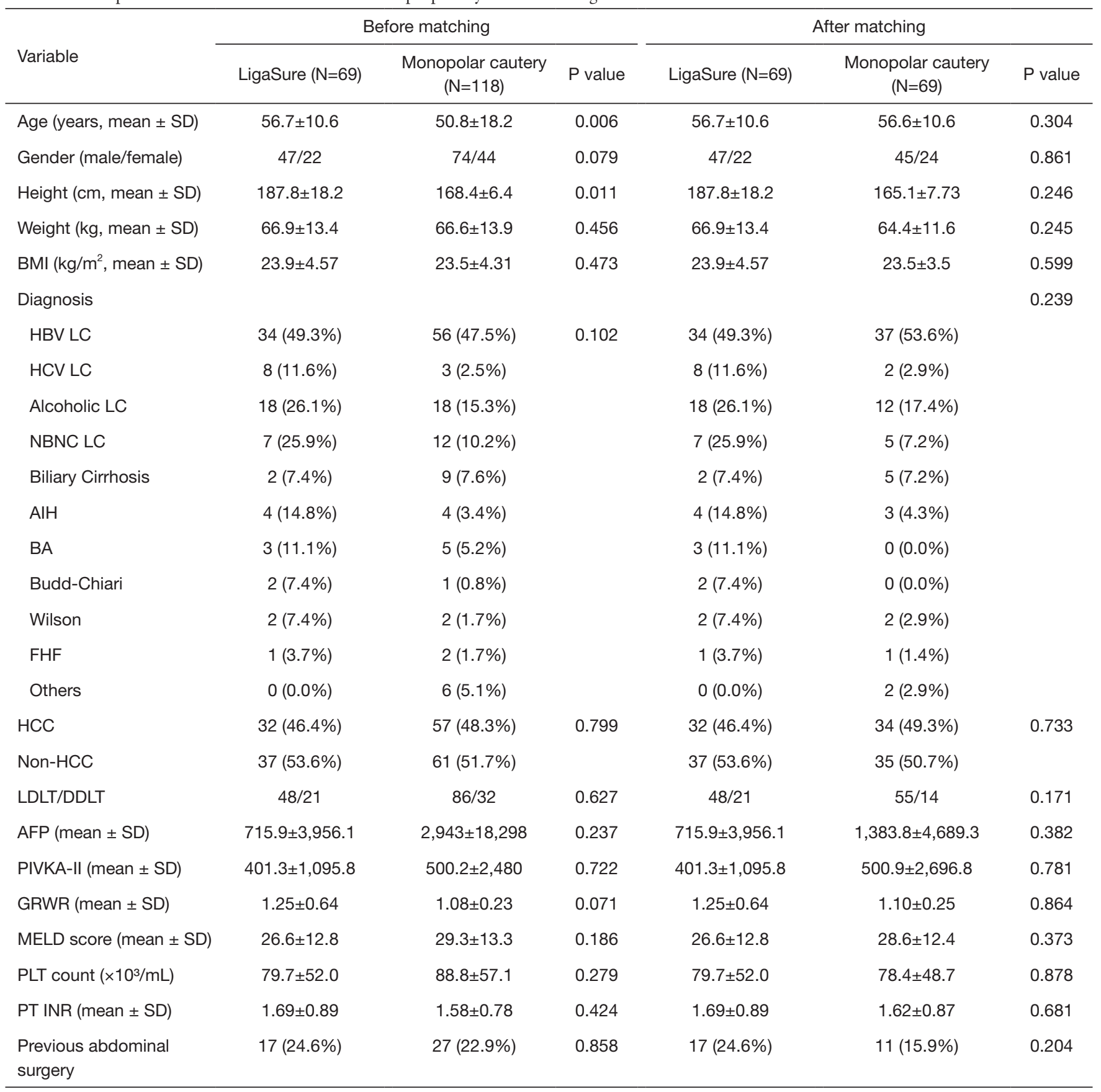

BMI, body-mass index; HBV LC, hepatitis B virus-related liver cirrhosis; HCV LC, hepatitis C virus-related liver cirrhosis; NBNC LC, non-B non C liver cirrhosis; AlH, autoimmune hepatitis; BA, biliary atresia; FHF, fulminant hepatic failure; HCC, hepatocellular carcinoma; LDLT, living donor liver transplantation; DDLT, deceased donor liver transplantation; AFP, alpha-feto protein; GRWR, graft recipient weight ratio; MELD, model for end-stage liver disease; PLT, platelet count; PT INR; prothrombin time international normalized ratio; PIVKA-II, protein induced by vitamin $\mathrm{K}$ absence II; PSM, propensity score matching; SD, standard deviation. 
Table 2 Comparison of the postoperative outcomes between the LigaSure group and the monopolar cautery group

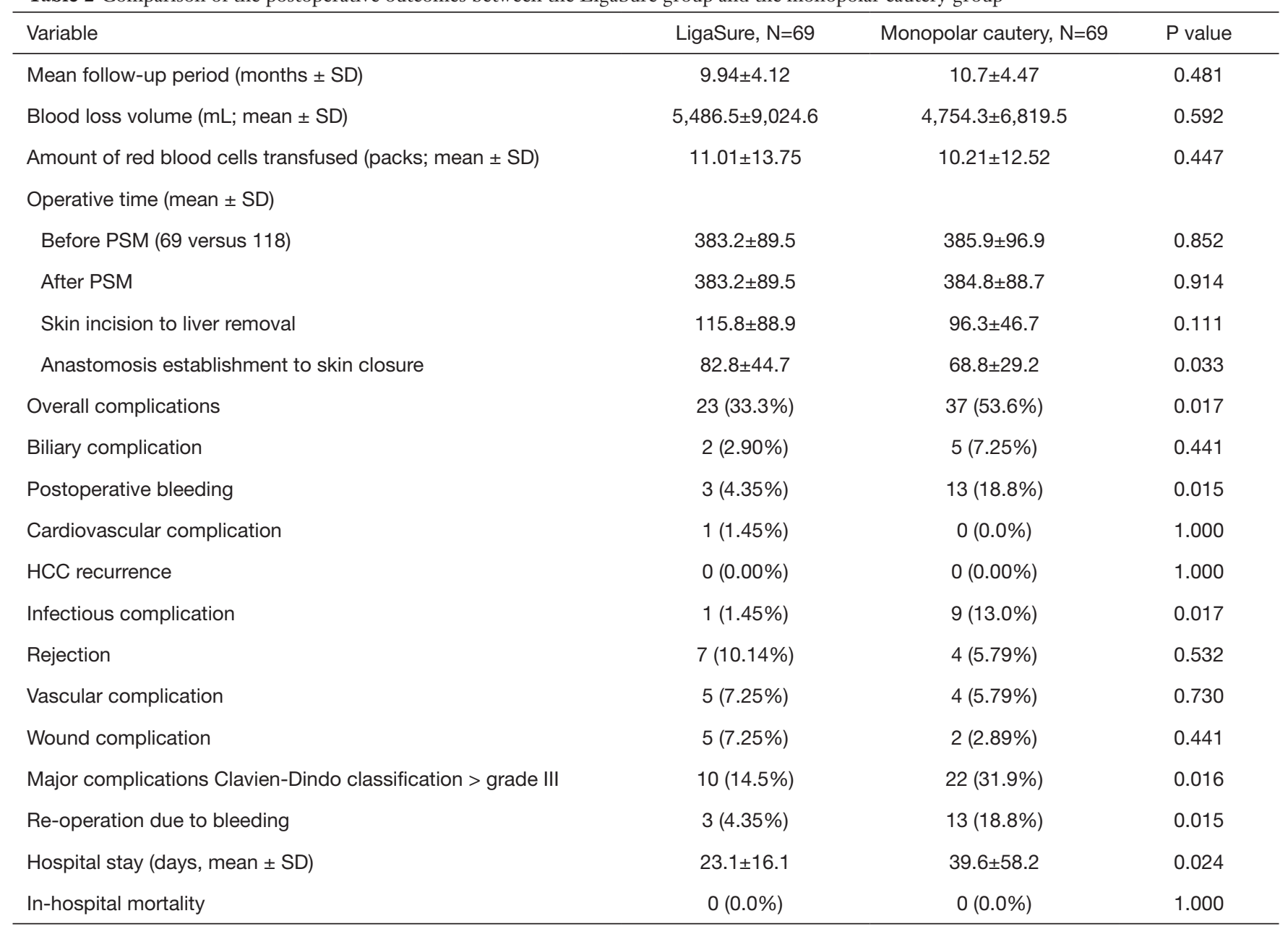

HCC, hepatocellular carcinoma; PSM, propensity score matching; SD, standard deviation.

group than in the monopolar cautery group (10, $14.5 \%$ versus $22,31.9 \% ; \mathrm{P}=0.016)$. The mean length of hospital stay was significantly less in the LigaSure group than in the monopolar cautery group (23.1 \pm 16.1 versus $39.6 \pm 58.2$ days; $\mathrm{P}=0.024)$. No significant differences were observed in the in-hospital mortality between the two groups.

\section{Discussion}

Over the last two decades, there has been significant progress in hepatectomy with the advancements in technology related to surgical instruments and techniques. The LigaSure energy device has significantly contributed to laparoscopic surgery to improve convenience and provide accurate sealing $(9,11,13,14)$. It is typically used as a device for liver parenchymal dissection during laparoscopic hepatectomy and tissue division to achieve liver mobilization. Therefore, several randomized controlled trials have been conducted to evaluate the clinical benefits of different liver transection methods in open liver resection. To date, several animal studies have been shown to compare the safety and effectiveness of Cavitron ultrasonic surgical aspirator (CUSA), LigaSure energy device, and ultrasonic shears for laparoscopic liver parenchymal transection $(17,20)$.

LT is frequently associated with significant blood loss and considerable transfusion requirements. Uncontrolled bleeding and catastrophic hemorrhages were some of the significant causes of perioperative mortality and morbidity. The intraoperative transfusion requirements may be a surrogate of liver cirrhosis severity and an indicator of technical difficulties during surgery; the outcomes are 
impacted by the severity of liver disease and technical problems. Other surgical factors related to high blood transfusion requirements during LT include previous upper abdominal surgery, portal vein thrombosis, and previous portoenterostomy.

There was no statistically significant difference in the mean operative time between the two groups. However, the energy device was generally not used in anastomosis procedures but was traditionally used during recipient hepatectomy. Furthermore, we analyzed the time to liver removal and bleeding control time following the anastomosis establishment. The time to liver removal was longer in the LigaSure group than in the monopolar cautery group; however, the difference was not statistically significant. Contrarily, the bleeding control time following the establishment of anastomosis was significantly longer in the LigaSure group than in the monopolar cautery group. It might be related to longer coagulation time in the LigaSure group (mean of 2-3 s) than in the monopolar cautery group.

No statistically significant difference was observed in intraoperative blood loss volume between the two groups. However, the rates of postoperative bleeding events and subsequent re-operation were significantly less in the LigaSure group than in the monopolar cautery group. Following surgery, bleeding was a problem in the areas of the triangular ligament and Gerota's fascia. Adequate coagulation can be achieved by monopolar cautery. Nevertheless, on average, hemostasis appears to be incomplete due to the high speed of monopolar cautery during liver removal. Contrarily, coagulation and mobilization using LigaSure were performed after sufficient heat application. Therefore, the rate of rebleeding is considered low due to adequate sealing.

A statistically significant difference was observed in the overall rate of surgical complications between the LigaSure group and monopolar cautery group. It seems that the high rate of re-operation caused the difference due to bleeding, and the rate of infectious complications after bleeding is likely to have influenced the incidence of infections and wounds $(8 / 13,61 \%)$. Thus, the length of hospital stay may have increased following surgery. However, no graft loss or patient death occurred due to surgical complications in both groups.

As in earlier studies, our results indicate that the reliability of ligation influences tumor recurrence during tumor resection and less blood loss (21), and recurrence detected during long-term follow-up assessments is prevented by implementing the no-touch technique (22).
We attempted to determine if the sealing effect caused HCC recurrence; however, the follow-up period was short, and there was no recurrence within the follow-up period. To make this determination, it is therefore essential to conduct studies with long follow-up periods.

This study has several limitations. It is not a randomized controlled trial, but it is a retrospective comparative study. And also, this study has a short follow-up period, and the sample size is small. However, PSM maximally corrects the factors that may influence postoperative bleeding and reoperation. And although there is still a randomized control study on the usefulness of energy devices in liver transplant surgery, there has not been any report on the results (12). It is also noteworthy that there are no previous studies on LT recipients expected to have massive bleeding that has reported a low rate of secondary bleeding that resulted in the reductions in the rate of secondary complications and length of hospital stay. In this study, the focus was on early postoperative outcomes; therefore, we could not report on the recurrence of HCC. If a follow-up study with a sufficiently long follow-up period was conducted, we could reach conclusions on the added advantages of energy devices concerning HCC recurrence.

In conclusion, in this study, we showed the possibility that the ligasure energy device can reduce postoperative bleeding in LT and reduce hospital stay by reducing secondary complications. However, a well-designed followup randomized control study is still needed to conclude on the merits of using energy devices in LT.

\section{Acknowledgments}

Funding: None.

\section{Footnote}

Reporting Checklist: The authors have completed the STROBE reporting checklist. Available at https://dx.doi. org/10.21037/atm-21-1318

Data Sharing Statement: Available at https://dx.doi. org/10.21037/atm-21-1318

Peer Review File: Available at https://dx.doi.org/10.21037/ atm-21-1318

Conflicts of Interest: All authors have completed the ICMJE uniform disclosure form (available at https://dx.doi. 
org/10.21037/atm-21-1318). The authors have no conflicts of interest to declare.

Ethical Statement: The authors are accountable for all aspects of the work in ensuring that questions related to the accuracy or integrity of any part of the work are appropriately investigated and resolved. This study protocol conformed to the ethical guidelines of the Declaration of Helsinki (as revised in 2013). The Institutional Review Board of Seoul National University Hospital approved this study (No.: 2101-001-1183) and individual consent for this retrospective analysis was waived.

Open Access Statement: This is an Open Access article distributed in accordance with the Creative Commons Attribution-NonCommercial-NoDerivs 4.0 International License (CC BY-NC-ND 4.0), which permits the noncommercial replication and distribution of the article with the strict proviso that no changes or edits are made and the original work is properly cited (including links to both the formal publication through the relevant DOI and the license). See: https://creativecommons.org/licenses/by-nc-nd/4.0/.

\section{References}

1. Mallet M, Rudler M, Thabut D. Variceal bleeding in cirrhotic patients. Gastroenterol Rep (Oxf) 2017;5:185-92.

2. Ewaga H, Keeffe EB, Dort J, et al. Liver transplantation for uncontrollable variceal bleeding. Am J Gastroenterol 1994;89:1823-6.

3. Roullet S, Millas E, Biais M, et al. Risk factors for bleeding and transfusion during orthotopic liver transplantation.

Ann Fr Anesth Reanim 2011;30:349-52.

4. Groenland TH, Porte RJ, Metselaar HJ. Liver transplantation and risk of bleeding. Curr Opin Organ Transplant 2007;12:287-93.

5. Figiel W, Grąt M, Wronka KM, et al. Reoperations for Intraabdominal Bleeding Following Deceased Donor Liver Transplantation. Pol Przegl Chir 2016;88:196-201.

6. Kloesel B, Kovatsis PG, Faraoni D, et al. Incidence and predictors of massive bleeding in children undergoing liver transplantation: A single-center retrospective analysis. Paediatr Anaesth 2017;27:718-25.

7. Ali A, Bhardwaj HL, Heuman DM, et al. Coronary events in patients undergoing orthotopic liver transplantation: perioperative evaluation and management. Clin Transplant 2013;27:E207-15.

8. Cleland S, Corredor C, Ye JJ, et al. Massive haemorrhage in liver transplantation: Consequences, prediction and management. World J Transplant 2016;6:291-305.

9. Giordano S, Kangas R, Veräjänkorva E, et al. Ligasure impact $^{\mathrm{TM}}$ might reduce blood loss, complications, and reoperation occurrence after abdominoplasty in massiveweight-loss patients: A Comparative Study. Scand J Surg 2020;109:151-8.

10. Roullet S, Freyburger G, Cruc M, et al. Management of bleeding and transfusion during liver transplantation before and after the introduction of a rotational thromboelastometry-based algorithm. Liver Transpl 2015;21:169-79.

11. Lee CL, Wu KY, Huang CY, et al. Comparison of LigaSure $^{\mathrm{TM}}$ tissue fusion system and a conventional bipolar device in hysterectomy via natural orifice transluminal endoscopic surgery (NOTES): A randomized controlled trial. Taiwan J Obstet Gynecol 2019;58:128-32.

12. Houben $P$, Khajeh E, Hinz U, et al. SEALIVE: the use of technical vessel-sealing devices for recipient hepatectomy in liver transplantation: study protocol for a randomized controlled trial. Trials 2018;19:380.

13. Levine NL, Zhang Y, Hoang BH, et al. LigaSure Use Decreases Intraoperative Blood Loss Volume and Blood Transfusion Volume in Sarcoma Surgery. J Am Acad Orthop Surg 2019;27:841-7.

14. Amirkazem VS, Malihe K. Randomized clinical trial of ligasure ${ }^{\mathrm{TM}}$ versus conventional splenectomy for injured spleen in blunt abdominal trauma. Int J Surg 2017;38:48-51.

15. Upadhyaya A, Hu T, Meng Z, et al. Harmonic versus LigaSure hemostasis technique in thyroid surgery: A metaanalysis. Biomed Rep 2016;5:221-7.

16. Fujita J, Takiguchi S, Nishikawa K, et al. Randomized controlled trial of the LigaSure vessel sealing system versus conventional open gastrectomy for gastric cancer. Surg Today 2014;44:1723-9.

17. Sellier C, Carabalona J, Hahn H, et al. Use of a cavitron ultrasonic surgical aspirator for parenchyma-sparing and complex liver resections in dogs. Vet Surg 2020;49:800-10.

18. Liu F, Wei Y, Li H, et al. LigaSure versus CUSA for parenchymal transection during laparoscopic hepatectomy in hepatocellular carcinoma patients with cirrhosis: a propensity score-matched analysis. Surg Endosc 2018;32:2454-65.

19. Lamattina JC, Hosseini M, Fayek SA, et al. Efficiency of the LigaSure vessel sealing system for recipient hepatectomy in liver transplantation. Transplant Proc 2013;45:1931-3. 
20. Öhlund M, Höglund O, Olsson U, et al. Laparoscopic ovariectomy in dogs: a comparison of the LigaSure ${ }^{\mathrm{TM}}$ and the SonoSurg ${ }^{\mathrm{TM}}$ systems. J Small Anim Pract 2011;52:290-4.

21. Katz SC, Shia J, Liau KH, et al. Operative blood loss independently predicts recurrence and survival after resection of hepatocellular carcinoma. Ann Surg

Cite this article as: Lee JM, Hong K, Han ES, Suh S, Hong S, Hong SK, Choi Y, Yi NJ, Lee KW, Suh KS. LigaSure versus monopolar cautery for recipient hepatectomy in liver transplantation: a propensity score-matched analysis. Ann Transl Med 2021;9(13):1050. doi: 10.21037/atm-21-1318
2009;249:617-23.

22. Lee JM, Lee KW, Kim HC, et al. No touch isolation technique for the prevention of postoperative recurrence of hepatocellular carcinoma after liver transplantationcombined with trans-arterial radioembolization. Surg Oncol 2020;35:189-90. 